\title{
NASA Research and Analysis: Status, Issues, and Recommendations for the Planetary Science and Astrobiology Decadal Survey Committee
}

\author{
Julie Castillo-Rogez ${ }^{1}$, Mark Sykes ${ }^{2}$, Amanda Hendrix ${ }^{2}$, \\ Julie Rathbun ${ }^{2}$, Christina Richey ${ }^{1}$, Paul K. Byrne ${ }^{3}$
}

\author{
Cosigners/Endorsers \\ Fayolle ${ }^{1}$, Tuan H. $\mathrm{Vu}^{1}$, Lori K Fenton ${ }^{5}$, Matija $\mathrm{Cuk}^{5}$ \\ 1- Jet Propulsion Laboratory, California Institute of Technology, Pasadena, CA. \\ 2- Planetary Science Institute, Tucson, AZ, \\ 3- North Carolina State University, Raleigh, NC. \\ 4- Lunar and Planetary Laboratory, University of Arizona, Tucson, AZ/ \\ 5- SETI Institute, Mountain View, CA.
}

James T. Keane ${ }^{1}$, Jessica J. Barnes ${ }^{4}$, Cynthia B. Phillips ${ }^{1}$, Paul V. Johnson ${ }^{1}$, Mathieu Choukroun ${ }^{1}$, Edith

\section{Acknowledgements}

Part of this research was carried out at the Jet Propulsion Laboratory, California Institute of Technology, under a contract with the National Aeronautics and Space Administration. Pre-Decisional Information - For Planning and Discussion Purposes Only. 


\section{EXECUTIVE SUMMARY}

This white paper is motivated by the very low and continuously declining selection rates ( $20 \%)$ of the PSD research and analysis programs over the past three to five years. Changes that would ensure a robust, sustainable review and proposal system are addressed in the white paper by Byrne et al.

\section{Key findings}

- NASA PSD R\&A funding lacks transparency, especially the way in which funding is allocated from the different budget lines available to NASA.

- While the planetary community generally considers the Planetary Science Division (PSD) Research and Analysis (R\&A) to be openly competed fundamental science and data analysis programs in the ROSES call, there is significant variation in what NASA has considered R\&A; this difference of perception resulted in budget profiles for these programs varying by more than $50 \%$ in separate 2017 submissions to the midterm Decadal Survey review panel [Sykes et al. 2017]. This discrepancy was in part due to the second planetary Decadal Survey ("Vision and Voyages") not clearly and specifically defining what to include in the term "R\&A."

- The Internal Scientist Funding Model (ISFM), i.e., research packages using R\&A funds and allocated to civil servant centers without competition with the rest of the community, has significantly altered the amount of openly competed R\&A funding.

- The PSD R\&A program is critically underfunded to the detriment of NASA's strategic objectives. Budget analysis [Sykes 2017] suggests that NASA PSD was not compliant with the second Decadal's recommendation regarding R\&A funding levels and their priority in the context of overall insufficient funds available to the Division. Since the last Decadal Survey, the field has grown substantially, with an increased need for more R\&A funds; proposal requests are also increasing (see below).

- The selection rates in the Planetary Science Division remain the lowest across all of NASA SMD's divisions, including Heliophysics and Earth Science, and cross-divisional programs.

- Unsustainable selection rates (i.e., <30\%) and the $2014 \mathrm{R} \& \mathrm{~A}$ reorganization are leading to more complex and expensive proposals and more effort dedicated to proposal writing because the budget allocations to key programs have not increased commensurately. The current scenario, if unchecked, risks depleting the ranks of new scientists and depriving them of the mid-career and senior scientists needed to mentor them and integrate them effectively into missions and broad collaborative efforts.

- The lack of commitment by NASA to the stability and sustainability of R\&A programs is a major source of stress and leads the community to spend a significant time writing proposals, sometimes at a major personal cost (see White Paper by 
Vance et al.). This scenario is not sustainable for a healthy and thriving planetary science community.

- The prospect of the community losing science experts and innovators (especially those without tenure/hard money) is a very real risk.

- The current system lacks transparency on what is meant by programmatic balance and how it is achieved when so few proposals are selected.

- Rathbun et al. (2020) point out that low selection rates disproportionately affect members of underrepresented groups, resulting in a less diverse and inclusive workforce.

- The formation of the Solar System Workings program was contrary to the suggestion of the latest Decadal to "merge related research programs" given that the programs merged to form it were not all related.

\section{Recommendations}

- R\&A needs to be clearly defined by the Decadal Survey and NASA must be transparent in its spending on every $R \& A$ program over the next decade. ISFM should be funded separately from R\&A. How programmatic balance is achieved in the context of low selection rates should be addressed by NASA and publicly communicated.

- The budget for R\&A, including a breakdown by programs, used to be regularly presented at assessment group meetings, as well as at large planetary conferences. However, this practice stopped in $2017-2018$. This communication to the community should be reinstated and include a clear description of funding lines for research and analysis, data analysis and participating scientist programs, and instrument programs.

- NASA PSD should support a FY23 R\&A budget of $\$ 500 \mathrm{M}$, to grow $1.5 \%$ above inflation each year for the rest of the decade. NASA should support a "healthy R\&A program" that does not unfairly penalize some sectors of the community over others (e.g., soft money scientists, minoritized persons, early career, etc.) and thus prevent the loss of expertise or represent a barrier to early career scientists.

- Solar System Workings should be broken up into at least five core programs of more target/discipline focus (see Sykes et al. White Paper).

- NASA PSD should carry out an assessment of the rate of infusion of instruments and other technologies competed via ROSES in order to evaluate the efficacy of these programs. 


\section{STATE OF NASA PSD "RESEARCH AND ANALYSIS"}

"Research and Analysis" currently lacks a proper definition consistent throughout the years: see Sykes et al. ("NASA Planetary Research and Analysis: What is R\&A?" White Paper). In brief, R\&A should encompass basic science, data analysis of past and current missions, and laboratory support. R\&A should not fund technology development. Having a consistent definition, used both by the Decadal Survey and by NASA, will help to ensure compliance with Decadal recommendations throughout the next decade.

NASA missions should adequately fund mission team members to conduct science investigations using mission data, and should also adequately fund Participating Scientist programs (see White Paper by Prockter et al.) NASA R\&A programs, specifically Data Analysis Programs, should adequately fund analysis of current and past mission data, particularly by non-team-members. Such programs enable broadening of the science data obtained by the mission and also allow for extension of data usage beyond the mission team. All interested scientists should be able to use mission data, via DAPs, not just mission team members and participating scientists.

A healthy R\&A program should support sufficient subject matter expertise to support the wide range of solar system strategic objectives of the Planetary Science Division. The diversity and complexity of the science being done at present is high with respect to what was being done even a decade ago. In particular, R\&A funding needs to grow with the broadening range of investigations and increasing diversity of objects targeted by space missions. Until such time that NASA no longer seeks to explore everywhere in the solar system and beyond, the agency's growing planetary science portfolio will require a growing workforce. Diversity of expertise includes developing theoretical framework and experimental research for interpreting data returned by past/ongoing missions, and for preparing for future missions and pursuing the analysis of mission data. Experimental research about acquiring material properties and simulating processes in conditions relevant to various classes of targets is paramount for the formulation and planning of future missions. This kind of research requires sustained funding for the development and maintenance of complex facilities.

Experimental research also generally leads to more expensive proposals due to hardware and supplies purchase, as well as the need for laboratory personnel, additional costs incurred by safety protocols, and sometimes access to analytical facilities. Furthermore, for experimental research to lead to meaningful results, proposals often need to involve a diverse team, including theoreticians who apply the planned experimental results in models and data analysis. This leads to the kind of proposals that require an interdisciplinary team, in turn driving up the expense of proposals. The reorganization of PSD R\&A programs was intended to foster more interdisciplinary research instead of the more focused programs that existed prior to the 2014 reorganization. In response, the budget of SSW proposals, for example, has been increasing in 
cost more than the average proposals to focused programs (Figure 3). However, the SSW budget for new proposals has remained about constant and started decreasing in 2019.

\section{State of $R \& A$ Funding}

The state of R\&A funding is not well established per lack of a proper definition of what R\&A is and its funding lines (see_Sykes et al. White Paper) In 2017, during discussions of the National Academies Committee on the Review of Progress Toward Implementing the Decadal Survey Vision and Voyages for Planetary Sciences, the problems that have resulted in the last decade from the lack of a clear definition of 'R\&A,' and the lack of transparency over the decade by NASA in its spending, were demonstrated [Sykes 2017]. An estimation of appropriate selection rate and support is suggested in Section 2 of this document.

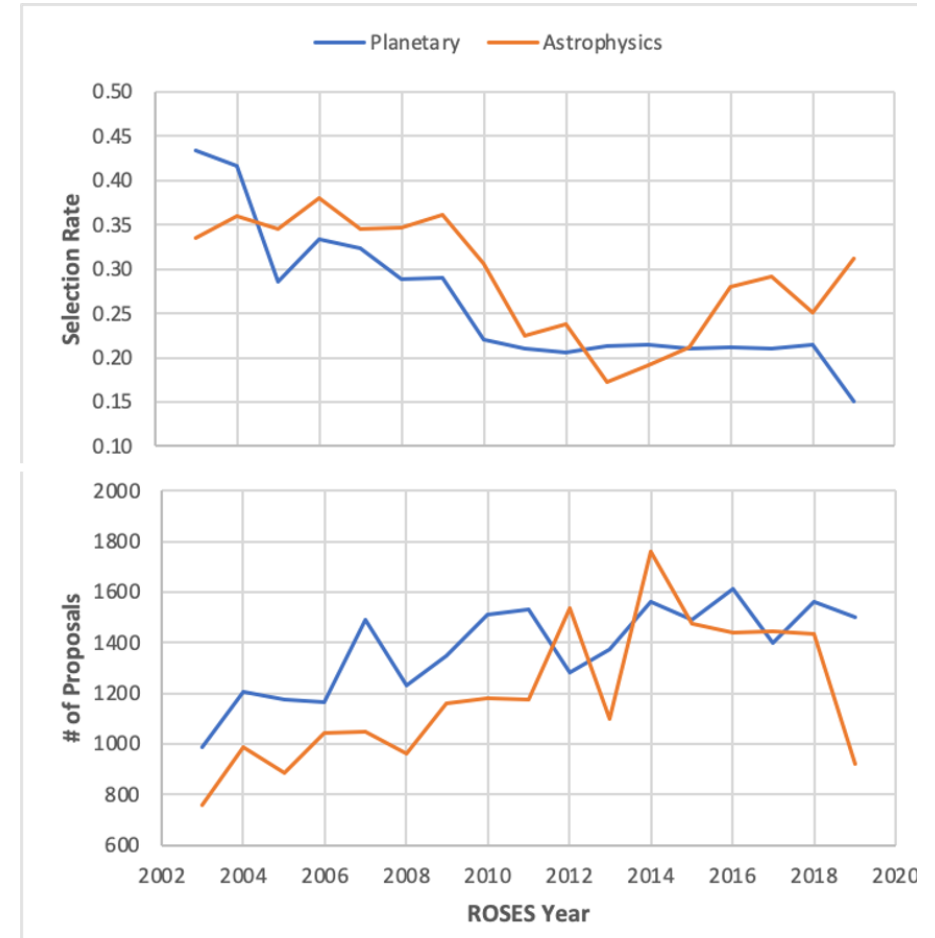

Figure 1. Number of proposals submitted to ROSES programs under the Planetary Science and Astrophysics Divisions between 2003-2019 (Source: SARA). Bottom chart shows that the number of proposals has remained between 1400-1600 over the last decadal. Variations from one year to the other reflects additional one-time calls (e.g., Participating Scientist Programs).

Top chart shows the selection rate has remained constant at $\sim 20 \%$ for the past decade. However, it dropped to $\sim 15 \%$ (averaged across all programs) in 2019. The low rate reflects a drop in, e.g., SSW $11 \%$ in 2019 vs. $\sim 20 \%$ in previous years) and PSTAR ( $4 \%$ in 2019 vs. $>10 \%$ in previous years). For clarity, we are showing only Planetary and Astrophysics, but Planetary remains the lowest selection rate across all of NASA SMD's divisions, including Heliophysics and Earth Science, as well as cross-divisional programs.

PSD's R\&A average selection rates have remained constant at $\sim 20 \%$ over the past decade, a drop from $\sim 30 \%$ in the previous decade. The average selection rate in 2019 was further down to $15 \%$ (Figure 1). Increases in the number proposed tasks, complexity, and overall proposal cost put further pressure on successful proposals. Recent disclosures regarding selection rates (e.g., the SSW rates at the August 2020 PAC meeting) imply that the current pressure on the community 
will continue and likely worsen. On the other hand, the selection rates for Astrophysics ROSES proposals have been consistently increasing since 2012 .

Frequent inconsistent reviews for proposals from one year to the next highlights the ineffectiveness of the review process. These aspects are addressed in a companion white paper by Byrne et al. Furthermore, Rathbun et al. show that low selection rates disproportionately affect members of underrepresented groups.

Data analysis programs are being used as substitutes for poorly funded extended missions, especially in the case of Discovery (see White Paper by Daubar et al.). Under the current paradigm, Phase F science funding is primarily intended for low-level data processing and archiving but is too short and scarce to permit the production of higher data products and analysis (i.e., Level 3 and 4). As a result, most investigators are left to seek funding for the publication of extended mission results via the data analysis programs, despite their significant involvement in science planning and operations.

The decreased support to R\&A is leading to cuts or reorganization of programs. For example, the Planetary Science Division in September 2020 elected to change the Habitable World and PSTAR programs to a biennial cadence as a way to alleviate funding shortfalls. Without clear explanation to the community, this choice appears arbitrary and could significantly affect members of the community whose research is primarily focusing on astrobiology, creating a barrier to early-career scientists.

Support for analytical facilities has also been decreasing, with the risk that the research infrastructure for planetary science will decay over the next decade and NASA losing the capability for example to fully study returned samples. More detail can be found in this NAS report and this white paper by Stroud et al. for CAPTEM that recommends NASA invest more heavily in analytical infrastructure as well as supporting laboratory personnel.
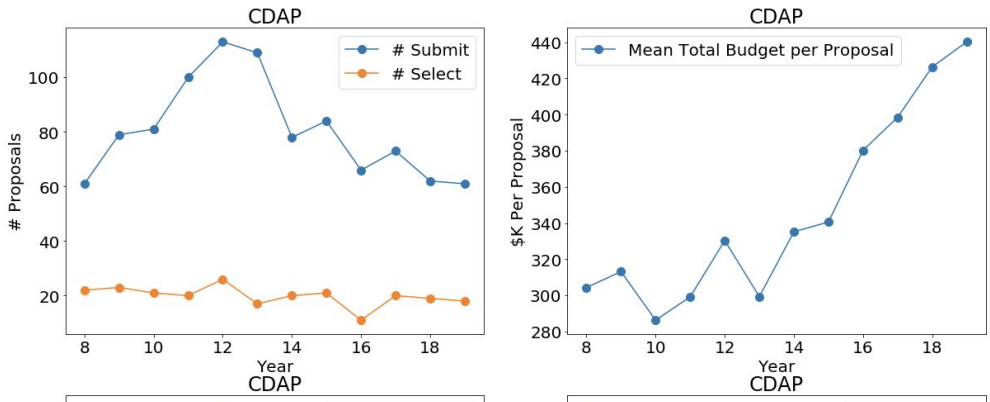

Figure 2. State of the Cassini Data Analysis Program since 2008 (Source. Throop, presentation to the Outer Planet Assessment Group, September 2020). Note that these are raw budget data not adjusted for inflation. These charts show
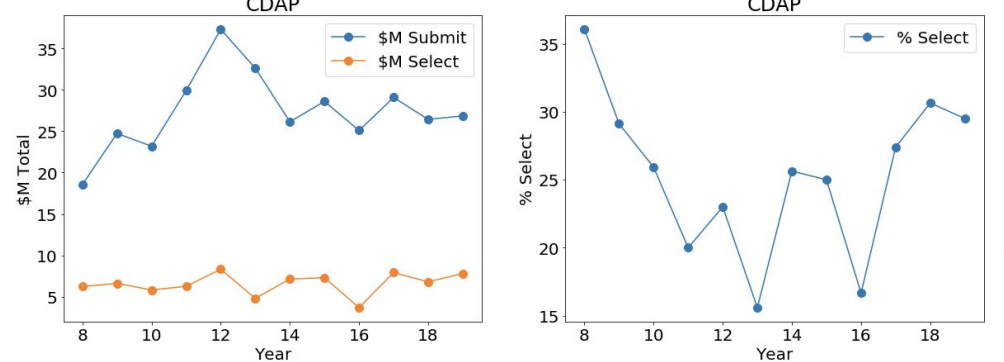
that the average proposal budget has increased by more than $40 \%$ since 2010, but the funding allocation to that program has shown large variations from one year to another. 
Figure 3. State of the SSW Program since 2008 (Source. Throop, presentation to the Outer Planet
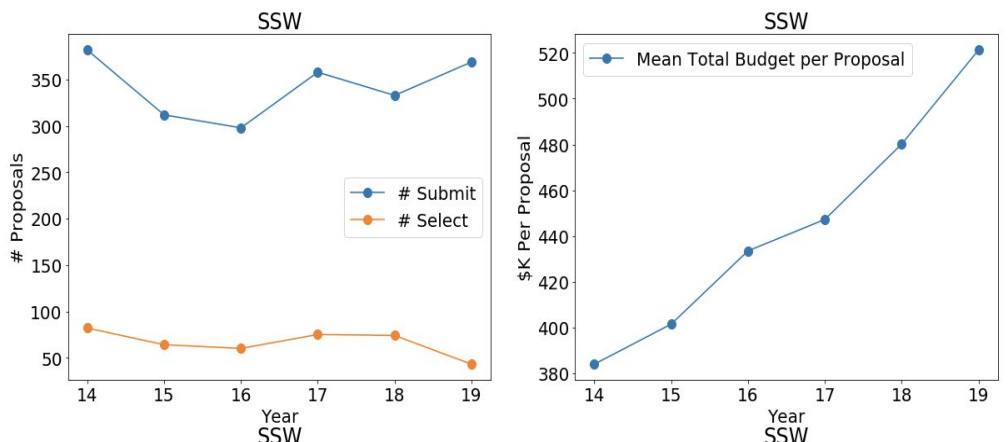

Assessment Group, September 2020).
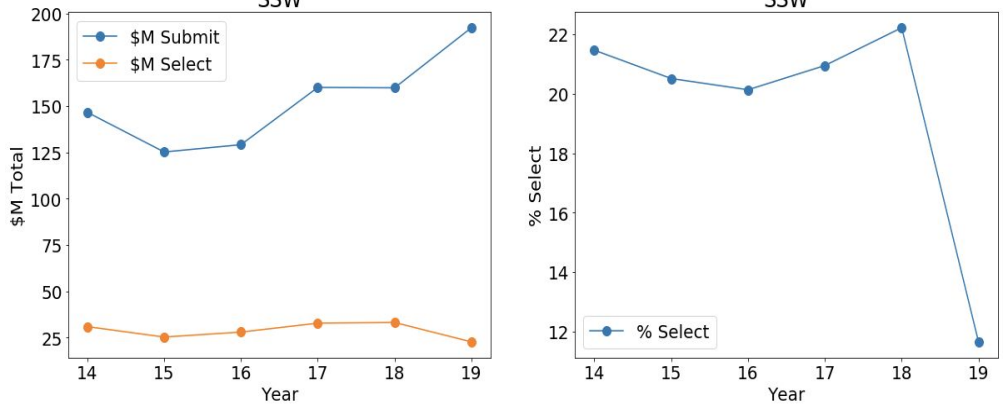

Note that these are raw budget data not adjusted for inflation.

Similarly to CDAP, the average proposal size has increased by $37 \%$ between 2014 and 2019 (vs. 8\% inflation). However, the budget has remained about constant while the total budget request for SSW has gone up from $\$ 125 \mathrm{M}$ to $\$ 192 \mathrm{M}(53 \%)$. As a result, the 2019 selection has been less than $12 \%$. It was announced on September 2, 2020 that the ROSES 2020 SSW budget for new awards would be cut from $\$ 10 \mathrm{M}$ to $\$ 7.5 \mathrm{M}$.

\section{WHAT IS A SUSTAINABLE R\&A FUNDING LEVEL?}

The R\&A funding level has to be scaled with the need, and so the recommendation from the previous decade is a starting point (see Appendix). But it should also fold in the need to maintain a diverse community, especially in terms of demographics. Hendrix and Rathbun (DPS Survey White Paper) include a recommendation to increase selection rates to no less than $30 \%$. Here is input from the previous Decadal Surveys:

- 1st Decadal (p. 163): "The ratio of submitted to funded proposals is typically 3 to 1 , which - the Solar System Exploration Survey believes - is too high, since at this rate new proposals can rarely be funded." (This is a 33\% selection rate.)

- 2nd Decadal (p. 10-3): "Over the 7 fiscal years 2003-2009, an average of 37 percent of the grant proposals submitted to an average of 18 or 19 programs in NASA's Planetary Science Division have been supported. The success ratio is lower than desirable but the negative impact of the low success rate on the science community is magnified by the small grant policy; many researchers seeking support for themselves and/or their students must submit half a dozen proposals each year to make ends meet." (This is a $37 \%$ selection rate.) 


\section{Vision and Voyages for Planetary Science in the Decade 2013-2022}

"Over the 7 fiscal years 2003-2009, an average of 37 percent of the grant proposals submitted to an average of 18 or 19 programs in NASA's Planetary Science Division have been

supported. The success ratio is lower than desirable"

"The committee strongly encourage NASA to find ways (e.g., by merging related research programs and lengthening award periods) to increase average grant sizes and reduce the number of proposals that must be written, submitted, and reviewed by the community."

"NASA should periodically evaluate the strategic alignment and funding level of all its SRA programs to ensure they remain healthy and productive."

- Aspiring for a rate of $40 \%$ is not unreasonable given past decadal analyses. Given the significant expansion of targets and science that has occurred even over just the past ten years, the first Decadal Survey's 's recommendation is still apt: "The SSE [Solar System Exploration] Survey recommends an increase over the decade 2003-2013 in the funding for fundamental research and analysis programs at a rate above inflation to a level that is consistent with the augmented number of missions, amount of data, and diversity of objects studied."

Assuming Sykes (2017)'s calculation of $\$ 176.1 \mathrm{M}$ for R\&A in FY11, the FY23 budget should be $\$ 257 \mathrm{M}$ per the Decadal Survey recommendation (assuming the NASA inflation index). The R\&A budget that NASA should support in the next decade should allow the necessary selection rate of $>30 \%$ and support the level of science that needs to be done in the next decade. Furthermore, the R\&A budget should reflect the fact that the planetary field is growing and that with more missions come more data to analyze, and with improved technologies come more capabilities to analyze the data. Thus, the Decadal Survey should recommend a funding level of $\$ 500 \mathrm{M}$ for FY23, to grow 1.5\% above inflation each year for the rest of the decade. This reflects both the need to achieve a more appropriate selection rate while also taking into consideration the exploding diversity of solar system exploration science that continues today and shows no sign of abating. 